Article

\title{
Adsorption and Gas Separation of Molecules by Carbon Nanohorns
}

\author{
Silvina M. Gatica ${ }^{1, *}$, Anton Nekhai ${ }^{2,+}$ and Adam Scrivener ${ }^{3, \ddagger}$ \\ 1 Department of Physics and Astronomy, Howard University, 2355 Sixth Street NW, Washington, \\ DC 20059, USA \\ 2 Rensselaer Polytechnic Institute, 110 Eighth Street, Troy, NY 12180, USA; nekhaa@rpi.edu \\ 3 Department of Computer Science, University of Rochester, 500 Joseph C. Wilson Boulevard, Rochester, \\ NY 14627, USA; ascriven@u.rochester.edu \\ * Correspondence: sgatica@howard.edu; Tel.: +1-202-806-7665 \\ + Participant in the summer Research Experiences for Undergraduates (REU), National Science Foundation \\ (NSF) summer program, at Howard University (2015). \\ $\ddagger$ Participant in the summer Research Experiences for Undergraduates (REU), National Science Foundation \\ (NSF) summer program, at Howard University (2014).
}

Academic Editor: Saikat Talapatra

Received: 14 March 2016; Accepted: 12 May 2016; Published: 19 May 2016

\begin{abstract}
In this paper, we report the results of Monte Carlo simulations of the adsorption of neon, argon, methane and carbon dioxide in carbon nanohorns. We model the nanohorns as an array of carbon cones and obtained adsorption isotherms and isosteric heats. The main sites of adsorption are inside the cones and in the interstices between three cones. We also calculated the selectivity of carbon dioxide/methane, finding that nanohorns are a suitable substrate for gas separation. Our simulations are compared to available experimental data.
\end{abstract}

Keywords: adsorption; carbon nanohorns; carbon dioxide

\section{Introduction}

The investigation of adsorption phenomena has been a very exciting and successful scientific activity in the last half-century [1-9]. In recent years, a considerable amount of work has been devoted to investigate, theoretically and experimentally, the physical adsorption in bundles of carbon nanotubes and carbon nanohorns [10-21]. The separation of gas mixtures has become a widespread practice used in industry and society. Some examples of the applications of gas separation include: the purification of natural gas and air, the sequestration of $\mathrm{CO}, \mathrm{CO}_{2}$ and other harmful gases and the production of $\mathrm{H}_{2}$. Within the last decade, environmental efforts have focused heavily on the reduction of $\mathrm{CO}_{2}$ in the atmosphere. The motive for targeting $\mathrm{CO}_{2}$ is a result of its "greenhouse gas effect" and its contribution to global warming.

Procedures for the separation of gases have also gained wide attention due to the importance that $\mathrm{CO}$ and $\mathrm{H}_{2}$ have managed to play in society. $\mathrm{CO}$, for example, is a valuable material for the synthesis of a variety of chemicals. In many instances, synthesized $\mathrm{CO}$ contains several impurities, such as $\mathrm{CO}_{2}, \mathrm{~N}_{2}$, $\mathrm{H}_{2}, \mathrm{CH}_{4}$ and $\mathrm{H}_{2} \mathrm{O}$, which requires purification through gas separation methods. Furthermore, in the development of a hydrogen-based economy, the efficient production of $\mathrm{H}_{2}$ represents an issue for commercial applications. Regardless of the mechanism, $\mathrm{H}_{2}$ purification ultimately equates to a $\mathrm{CO}_{2}$ removal process. Within the arena of gas separation techniques, carbon nanohorns are a promising material.

"Physical adsorption" (physisorption) is a term applied to atoms or molecules that are weakly bound to surfaces. Physisorption has been extensively explored for more than half of a century because of interest in both potential applications and basic science. These applications include the 
separation of cryogenic gases, their storage and their use as surface characterization tools, such as the measurement of the surface area of porous media by nitrogen adsorption. The science of physisorption encompasses a wide variety of fundamental questions, including many related to phase transitions. Adsorption studies also serve a crucial purpose in fundamental and applied characterization of porous materials. In particular, nitrogen adsorption at $77 \mathrm{~K}$ is routinely used for the characterization of porous structure. In the case of substrates with pore dimensions $<0.5 \mathrm{~nm}$, activated diffusion may occur at $77 \mathrm{~K}$ [22], thereby preventing the observation of nitrogen uptake. In these circumstances, the adsorption of carbon dioxide at $273 \mathrm{~K}$ may be used as a routine measurement of micropore volume. Adsorption studies also increase the scientific community's understanding of substrates when they are correlated with experimental results. The properties of adsorbates are thus one of the aspects of nanomaterials that needs to be assessed to enable its applications and to help bring its use in new technologies closer.

Gas separation by adsorption can be accomplished by three basic physical mechanisms: equilibria, kinetics and steric effects [23]. Equilibrium mechanisms rely on the strength of attraction between gas molecules and their substrate, while kinetic mechanisms involve the differences in the adsorption and transport rates of a gas on and through its substrate. Steric mechanisms, on the other hand, depend on the incompatibility between the size or shape of the adsorbate gas molecules and the pores of the substrate. For instance, since $\mathrm{CO}_{2}$ is typically found in a mixture with gases of similar size (for example, $\mathrm{CH}_{4}$ and $\mathrm{H}_{2}$ ), steric separation is not effective. However, $\mathrm{CO}_{2}$ possesses the strongest, attractive interactions with many substrates. For example, the energy of interaction of $\mathrm{CO}_{2}$ with graphene is twice as strong as $\mathrm{CH}_{4}$ with graphene [24]. As a result, the equilibrium mechanism presents the most plausible strategy.

Adsorption selectivity in a binary mixture of components $i$ and $j$ is defined as:

$$
S_{i / j}=\frac{x_{i} / x_{j}}{y_{i} / y_{j}}
$$

where $x_{i}$ and $y_{i}$ are the molar concentrations of species $i$ in the adsorbed phase and vapor phase, respectively. The concentration in the adsorbed phase, $x_{i}$, can be obtained from Monte Carlo or molecular dynamics simulations.

We can also estimate the selectivity of mixtures by the commonly-used "Ideal Adsorbed Solution Theory" (IAST) [25]. In the IAST, the selectivity is calculated using the data of the adsorption of the pure components, thus being a simple approach. Unfortunately, it may not be accurate for some mixtures that differ strongly in size or polarity. However, Babarao et al. confirmed that the theory is indeed reliable in the case of mixtures $\mathrm{CO}_{2}+\mathrm{CH}_{4}$ in IRMOFs [26].

The specification of an appropriate adsorbent is the key for separation by adsorption. Although, currently, there are materials that are useful for gas separation, research in this area continues in order to optimize the performance of current materials and to examine a wider range of new sorbents. Many substrates have been used for this purpose, for example carbon nanotubes, activated carbons, zeolites and metallic organic frameworks.

Carbon nanohorns resemble short, wide, highly-defected single-walled nanotubes that end in a conical tip ("horns") [27]. In contrast to regular nanotubes that assemble into parallel bundles, nanohorns form spherical aggregates with the nanohorns arranged along radial directions. The center of these aggregates is, in most cases, hollow. The body of the nanohorn is more or less cylindrical, with typical lengths between 30 and $70 \mathrm{~nm}$ and with a diameter that varies irregularly along its length (typical nanohorn diameters are between 3 and $8 \mathrm{~nm}$ ). The diameters of these spherules are typically 80 to $100 \mathrm{~nm}$.

Using the simulation technique grand canonical Monte Carlo (GCMC), we obtained the adsorption isotherms of $\mathrm{Ne}, \mathrm{Ar}, \mathrm{CH}_{4}$ and $\mathrm{CO}_{2}$ in an array of carbon nanohorns. We estimated the selectivity for $\mathrm{CO}_{2}$ based on the IAST approximation. 
This article is organized as follows: In Section 2, we explain the method and the model. In Section 3, we describe our results, and in Section 4, we present our conclusions.

\section{The Model}

In the numerical study of adsorption, the following experiment is simulated: the substrate is exposed to a vapor at constant $P$ and $T$. After some time passes, long enough to reach equilibrium, the uptake is measured under those $P$ and $T$ conditions. This can be simulated numerically by the methods of Grand Canonical Monte Carlo (GCMC) or Molecular Dynamics (MD) [28].

The GCMC method is based on statistical mechanics theory. The uptake is obtained at any given temperature and the chemical potential of the adsorbate. The chemical potential of the adsorbate is identical to that of the vapor, since both are in thermal equilibrium, and the last one is related to the pressure of the vapor, through the equation of state.

The inputs the data of the simulation are the pressure of the vapor, the temperature and the intermolecular forces. The output data of the simulation are the average number of adsorbed atoms $(N)$, the averages of the total energy, the energy gas-surface $\left(E_{g s}\right)$ and the energy gas-gas $\left(E_{g g}\right)$. We also collect samples of the coordinates for each of the adsorbed atoms. For each single data point in the isotherms $N(P, T)$, we typically run $3 \times 10^{6} \mathrm{MC}$ moves to reach equilibrium, and $10^{6}$ moves are performed for data collection. The ratio of creation/deletion/translation moves is 0.40/0.40/0.20. For details on the method, we refer the reader to [18].

Carbon nanohorns have two main sites of adsorption: inside the cones near the tip and in the external interstices between cones. We designed a simple version of the nanohorns, consisting of an array of seven "graphene" cones on the $8.0 \mathrm{~nm} \times 8.7 \mathrm{~nm}$ base of the simulation cell (see Figure 1). The cones are identical, with $2 \mathrm{~nm}$-diameter bases and 1.5-nm heights. Although the model lacks the spherical curvature typical of a dahlia-shaped arrangement, it holds the main features that are important for adsorption: interstices and conical tip. The boundary conditions are set reflective in the $z$ direction and periodic in $x, y$.

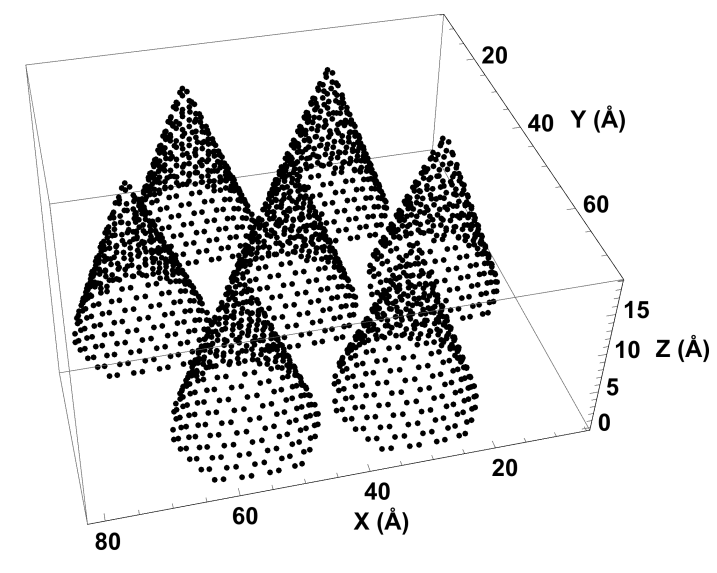

Figure 1. Simulation cell with seven nanohorns.

We compute the adsorption potential as the pairwise sum of two-body interactions between individual carbon atoms and the adatom,

$$
V(\vec{r})=\sum_{i} U\left(\left|\vec{r}-\vec{R}_{i}\right|\right)
$$

For Ar, Ne and $\mathrm{CH}_{4}$, we use the Lennard-Jones (LJ) potential to model the adsorbate-adsorbate and adsorbate-carbon interactions. 
The $\mathrm{CO}_{2}$ molecule was represented as a rigid three-site linear molecule with partial charges in each site $[29,30], q_{O}=-288 e$ and $q_{C}=+0.576 e$ and a bond length of $1.18 \AA$. These parameters were set to mimic the molecular quadrupole moment, $Q=-4.3 \mathrm{~B}$. The interaction energy between a $\mathrm{CO}_{2}$ molecule and each carbon atom in the nanohorn is the sum of LJ terms:

$$
U_{\mathrm{CO} 2-\mathrm{C}}=\sum_{i} U_{L J}^{i C}\left(r_{i C}\right)
$$

while the $\mathrm{CO}_{2}-\mathrm{CO}_{2}$ interaction is obtained as a sum of the $\mathrm{LJ}$ and electrostatic Coulomb terms:

$$
U_{\mathrm{CO} 2-\mathrm{CO} 2}=\sum_{i j}\left(U_{L J}^{i j}\left(r_{i j}\right)+k_{e} \frac{q_{i} q_{j}}{r_{i j}}\right)
$$

These sums are done over the carbon and oxygen atoms in the $\mathrm{CO}_{2}$ molecule.

The LJ parameters for the adatom-C potential are obtained by fitting the physical properties of the gases and using semi-empirical combining rules: $\sigma_{a C}=\left(\sigma_{a a}+\sigma_{C C}\right) / 2$ and $\epsilon_{a C}=\sqrt{\epsilon_{a a} \epsilon_{C C}}[31]$, with $\sigma_{C C}=3.4 \AA$ and $\epsilon_{C C}=28 \mathrm{~K}[17,32]$.

The LJ parameters are exhibit in Table 1.

Table 1. Lennard-Jones (LJ) parameters used in the simulations.

\begin{tabular}{ccc}
\hline Adatom & $\sigma(\AA)$ & $\epsilon(\mathbf{K})$ \\
\hline $\mathrm{Ar}[33]$ & 3.4 & 120 \\
$\mathrm{Ne}[33]$ & 2.75 & 35.6 \\
$\mathrm{CH}_{4}[34]$ & 3.7 & 148 \\
$\mathrm{C}$ in $\mathrm{CO}_{2}[29,30]$ & 2.8 & 29.7 \\
$\mathrm{O}$ in $\mathrm{CO}_{2}[29,30]$ & 3.0 & 83 \\
\hline
\end{tabular}

\section{Results}

The simulations were done for $\mathrm{Ne}, \mathrm{Ar}, \mathrm{CO}_{2}$ and $\mathrm{CH}_{4}$. The results are described below.

Neon: Figure 2 shows the adsorption isotherms for $\mathrm{Ne}$ at temperatures between $18.7 \mathrm{~K}$ and $49.8 \mathrm{~K}$, and Figure 3 shows the neon atoms in the simulation cell at four different $P, T$ conditions as labeled A to $\mathrm{D}$ in Figure 2. At the lowest coverage, the neon atoms are adsorbed in the most attractive sites, which are inside the cones (labeled $\mathrm{A}$ in the isotherms). At higher pressure, the adsorption occurs both inside and in the external interstices (label B). Adsorption continues to increase until the least attractive sites are populated, which are the walls of the cones opposite the interstices (label C), and finally, a "monolayer" forms covering all of the exterior of the cones (label D). The isotherms show a clear "bump" between C and D, similar to a monolayer completion usually seen in adsorption experiments, and another almost imperceptible bump at low coverage. The experiments reported in [19] show two bumps, as well. The pressure at which the condensation starts in the simulations is similar to the one in the experiment at the same temperature. However, in the simulations, the condensation step is less abrupt.

The quantum effects on the Ne isotherms are small. We conclude this after calculating the upper limit to both the Boer parameter $\Gamma$ and the zero-point energy (ZPE). Those correspond to the "worst-case scenario" in our simulations, that is $T=18.7 \mathrm{~K}$ and Ne-Ne separation $=\sigma_{L J}$, in which case, we obtain $\Gamma=0.3$ and $\mathrm{ZPE}=0.1 \mathrm{~K}$ (much smaller than the thermal, Ne-Ne and Ne-graphene energies). 


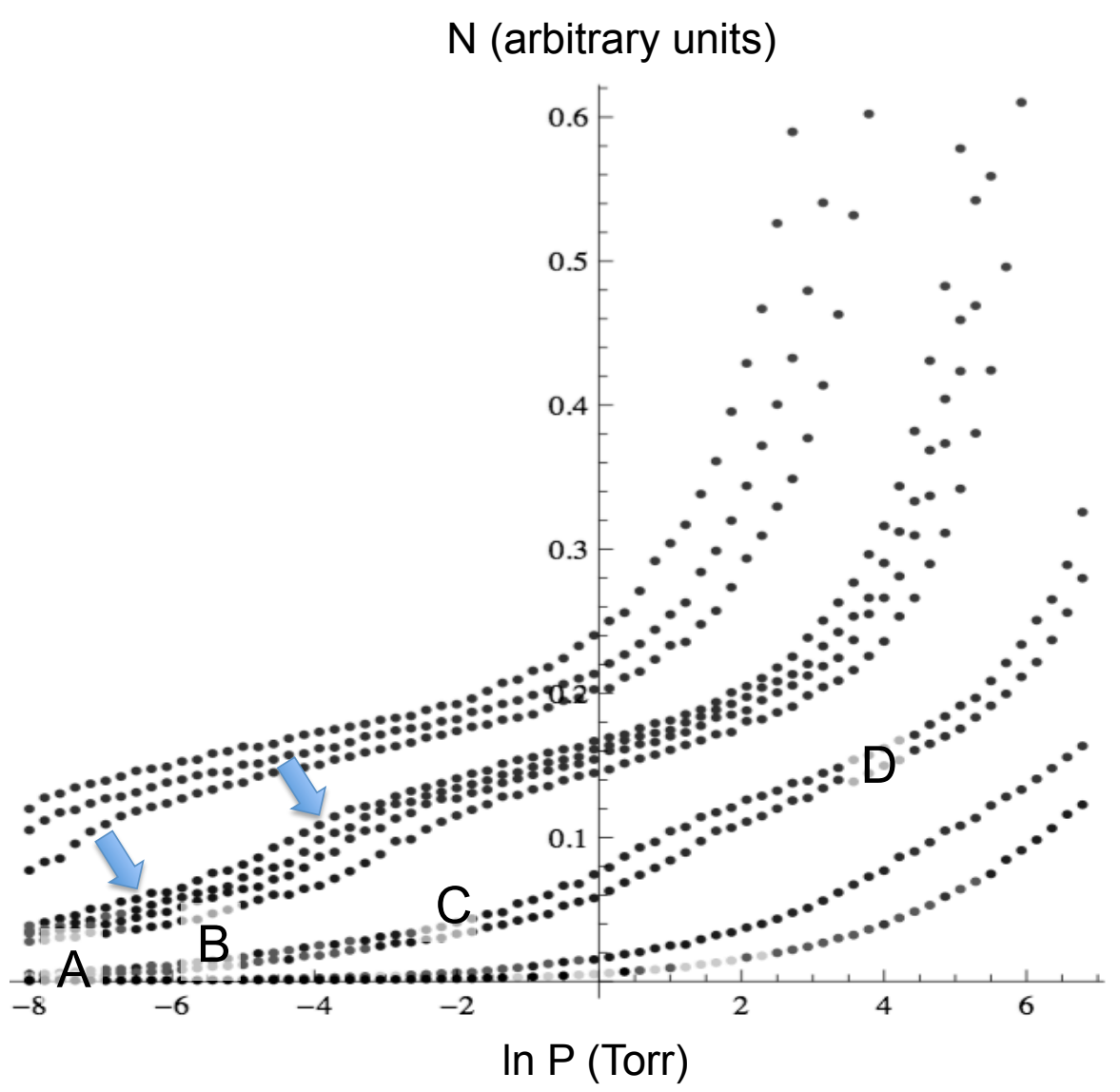

Figure 2. Adsorption isotherms for $\mathrm{Ne}$ at temperatures between $18.7 \mathrm{~K}$ and $49.8 \mathrm{~K}$. The arrows indicate the bumps, and the labels A to D correspond to the configurations shown in Figure 3.

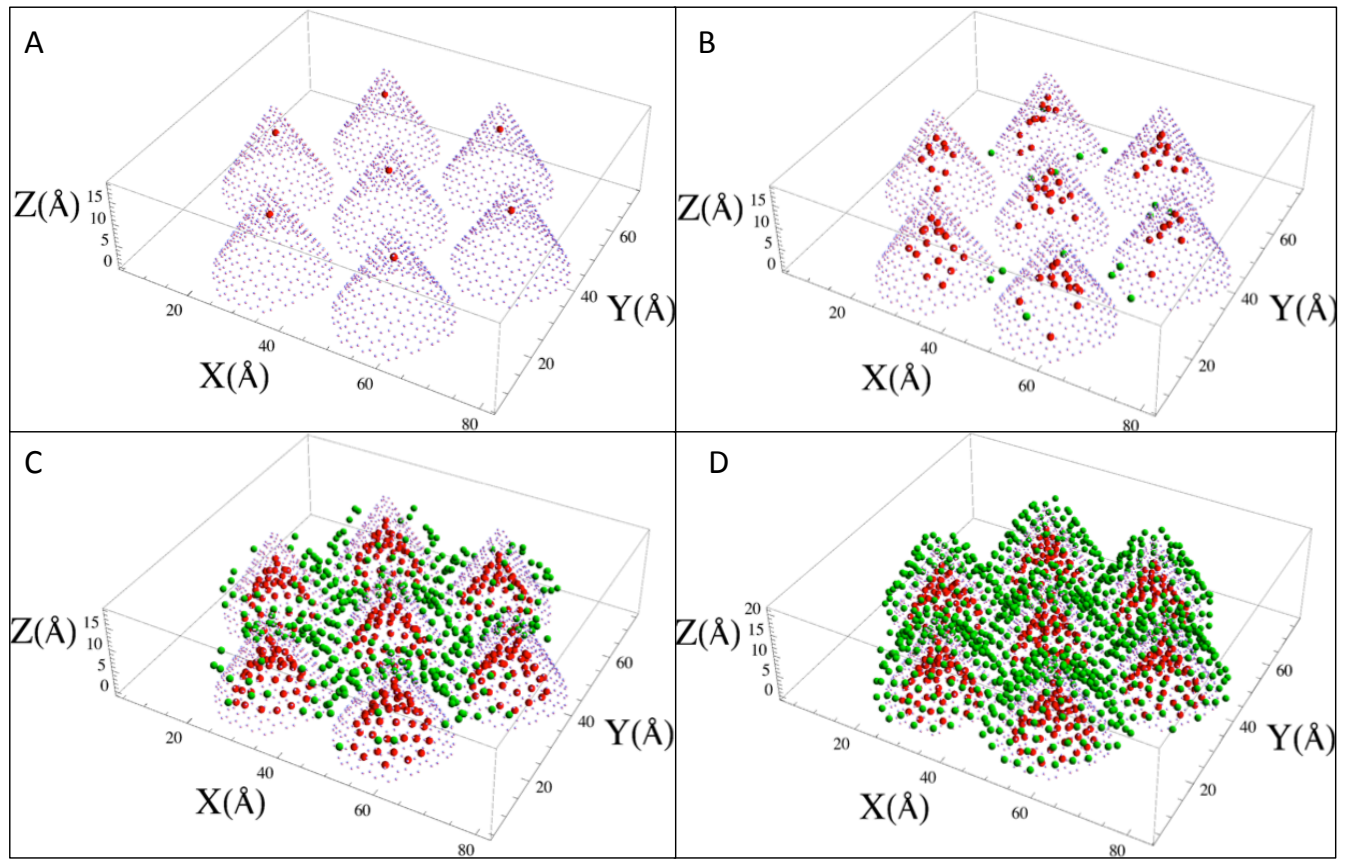

Figure 3. Sample configurations (at T, $\mathrm{P}$ conditions, indicated by the labels $\mathbf{A}$ to $\mathbf{D}$ in Figure 2 showing $\mathrm{Ne}$ atoms inside the cones (red) or outside (green). 
We computed the isosteric heat of adsorption from the isotherms, to compare to the experimental results from [19].

The isosteric heat of adsorption, $q_{s t}$, is defined as:

$$
q_{s t}(n, T)=k_{B} T^{2}\left(\frac{\partial \ln P}{\partial T}\right)_{n}
$$

where $n$ is the coverage and $P$ is the vapor pressure at the given $n, T$. We compute the $q_{s t}$ from the adsorption isotherms at a constant value of $n$. The result is displayed in Figure 4, which can be compared to the experimental result from [19].

We observe a plateau at approximately $20 \mathrm{meV}$, similar to the experimental value $(21 \mathrm{meV})$. However, the peak value in our simulations is higher than in the experiment $(37.5 \mathrm{meV})$. This indicates an overestimation of the adsorption energy inside the cones, probably an effect of the "pointy shape" of the cones.

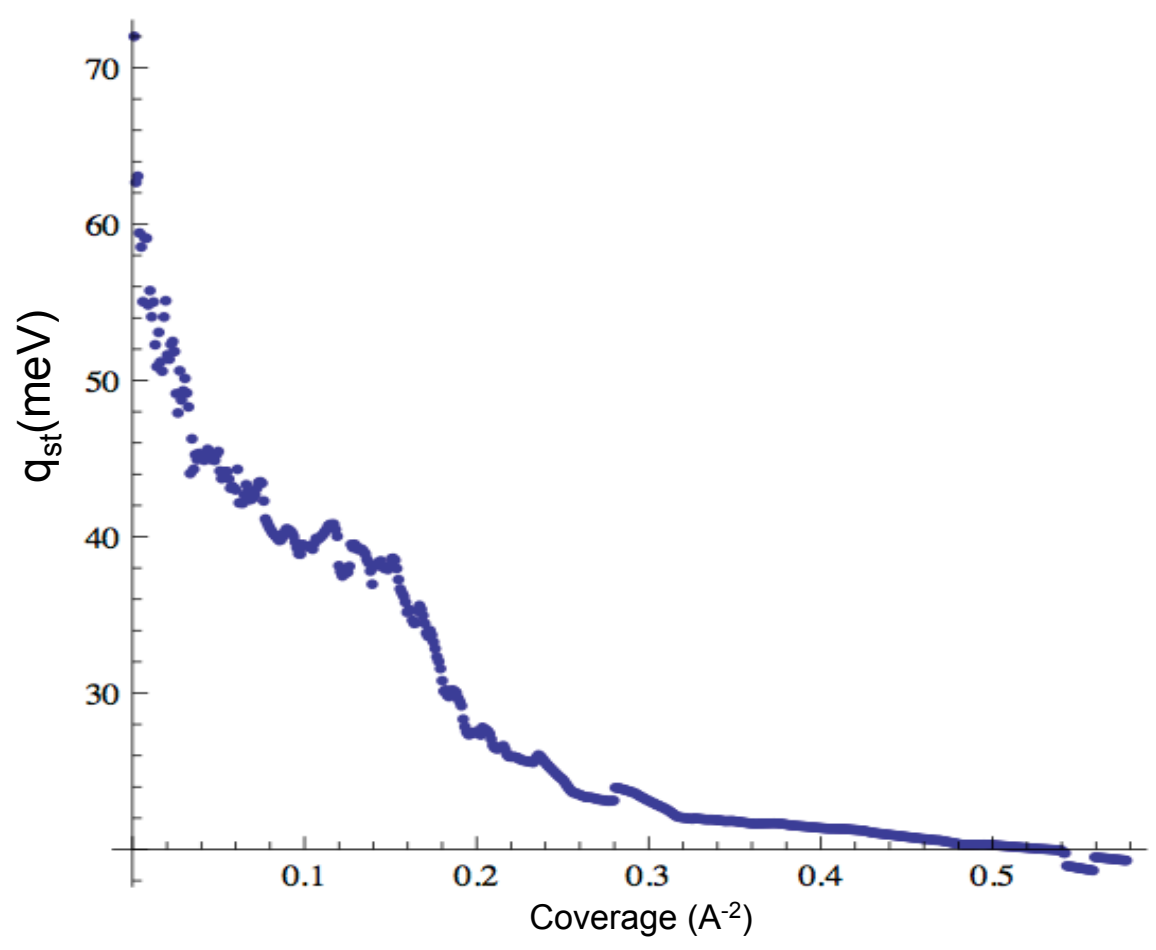

Figure 4. Isosteric heat of adsorption of Ne.

Argon: We simulated the adsorption of Argon at temperatures from 70 to $91 \mathrm{~K}$ in order to be able to compare to the available experimental data. Our results for Ar show similar features as $\mathrm{Ne}$ and reasonable agreement with the experimental results from Calvillo et al. [20]. We show the results in Figure 5. We identify two "bumps" at low coverage and medium coverage. In our simulations, the first bump corresponds to the occupancy of the interstices, and the second bump to the complete coverage of the external sites. We also see that the condensation in the cell starts at similar pressures as in the experiment [20]. The desorption isotherms show a slight hysteresis in the condensation regime. We also computed the isosteric heat of adsorption, shown in Figure 6. We find that the values of $q_{s t}$ obtained in our simulations for both adsorption and desorption are lower than the experimental $\mathrm{q}_{s t}[20]$. We believe that the reason for the discrepancy is the simplicity of our nanohorns. 

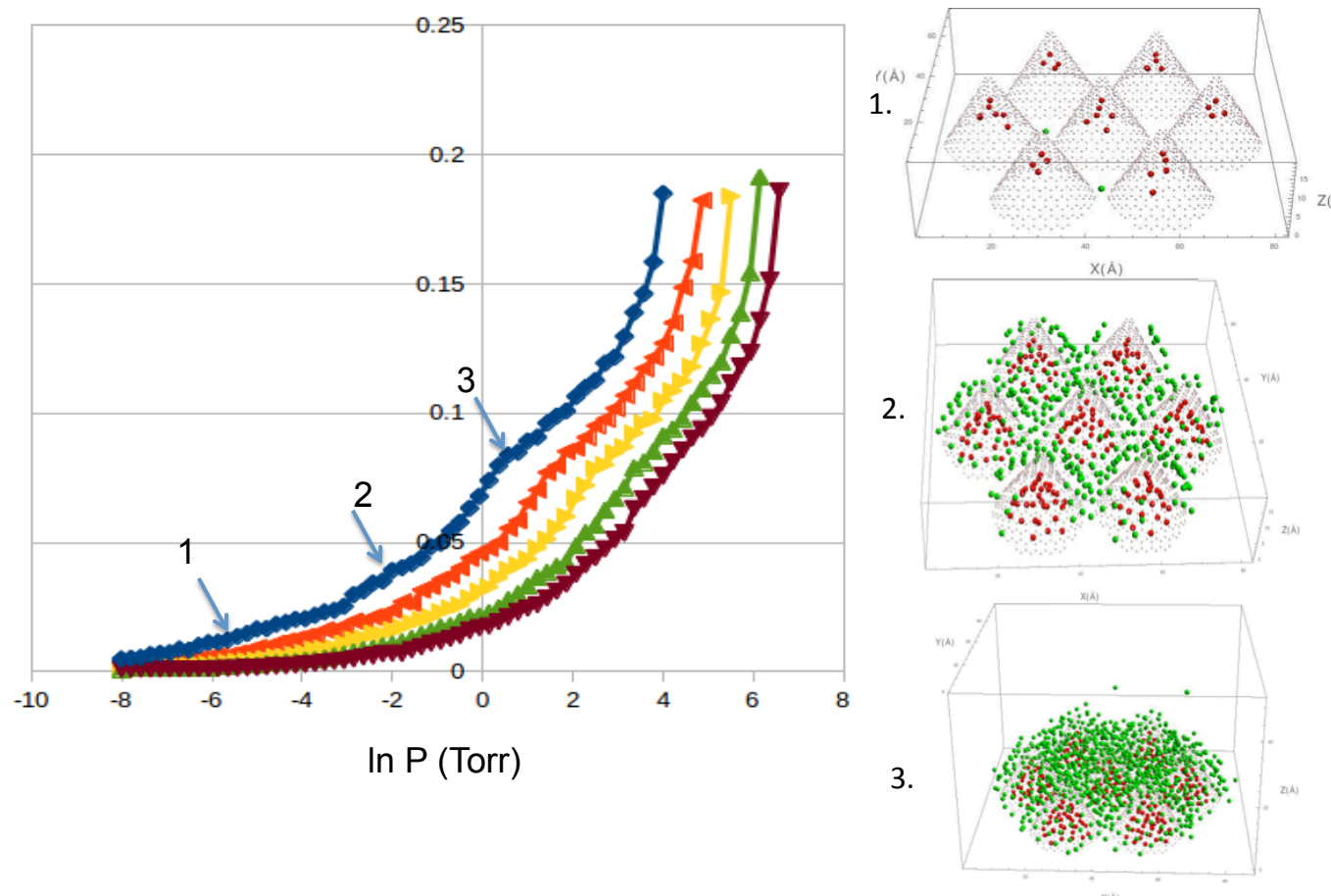

Figure 5. Adsorption isotherms of argon (in $\AA^{-2}$ ), at temperatures of $70.32,81.51,86.45$ and 91.13 $\mathrm{K}$ (from left to right). Also shown are configurations at low (1); medium (2) and high (3) coverage, as labeled. Color code: atoms inside the cones (red) and outside (green).

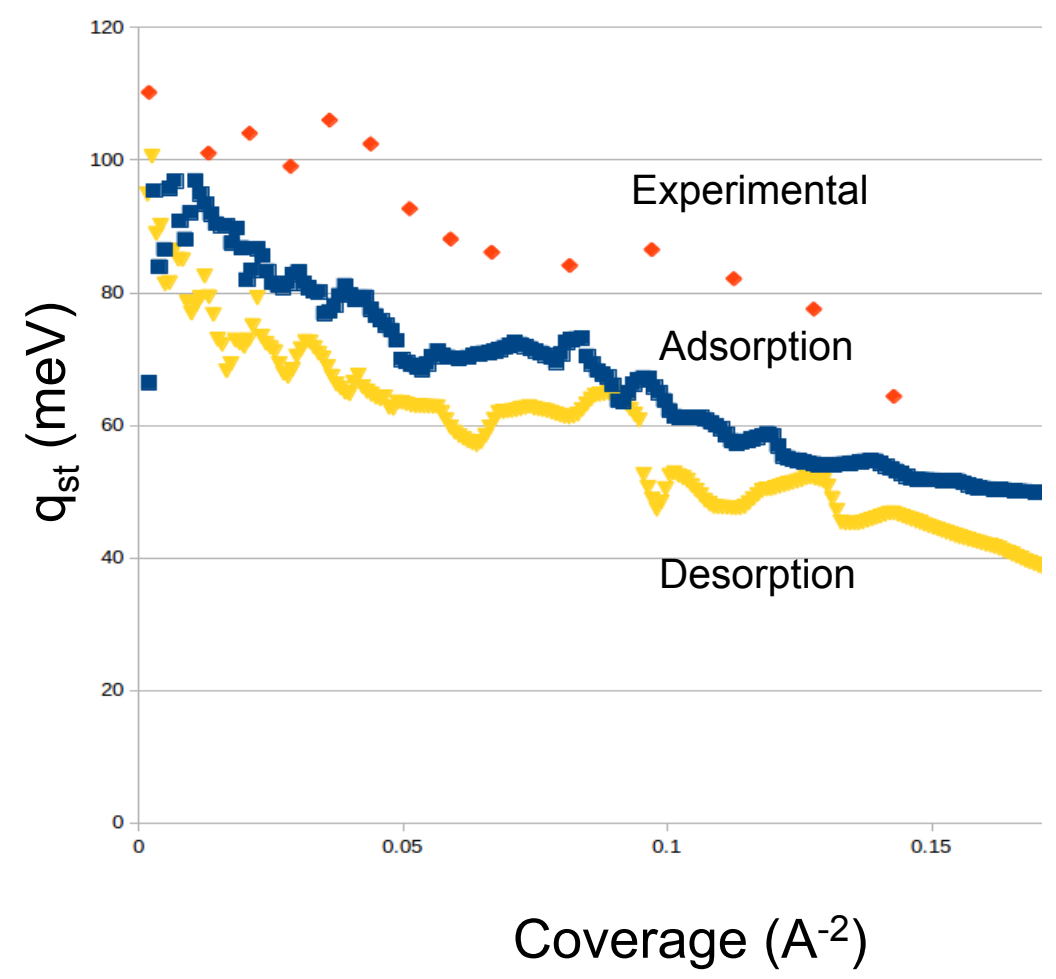

Figure 6. Isosteric heat of adsorption and desorption of argon. Also shown are the experimental data from [20]. 
$\mathrm{CO}_{2}$ and $\mathrm{CH}_{4}$ : In order to address the selectivity of mixtures, we run simulations for pure carbon dioxide and pure methane at temperatures from 143 to $193 \mathrm{~K}$ (see Figure 7). We found that $\mathrm{CO}_{2}$ is adsorbed at significantly lower pressure than $\mathrm{CH}_{4}$. As a result, the substrate adsorbs $\mathrm{CO}_{2}$ with high selectivity. From our isotherms and using the IAST approximation, we estimate the values of $\mathrm{S}_{\mathrm{CO} 2 / \mathrm{CH} 4}$ : $25,23,16,10,8$ and 6, for temperatures of $143 \mathrm{~K}, 150 \mathrm{~K}, 160 \mathrm{~K}, 173 \mathrm{~K}, 180 \mathrm{~K}$ and $193 \mathrm{~K}$, respectively.

We also obtained the isosteric heat of adsorption for $\mathrm{CO}_{2}$, which we display in Figure 8 for low to medium coverage. The low coverage limit is $230 \mathrm{meV}$, and the medium coverage value is near $140 \mathrm{meV}$. These values are lower than the experimental data, which range from 237 to $280 \mathrm{meV}$ [21]. However, we find agreement with the experiment in the fact that the isotherms for $\mathrm{CO}_{2}$ are smooth (no sub-steps or bumps), unlike the isotherms for Ar or Ne. The isosteric heat does not show a " $u$ " shape like the experimental $q_{s t}$ reported in [21]. In order to address this feature, we should obtain the values of the $q_{s t}$ at higher coverage, a regime in which we have no results to report yet.

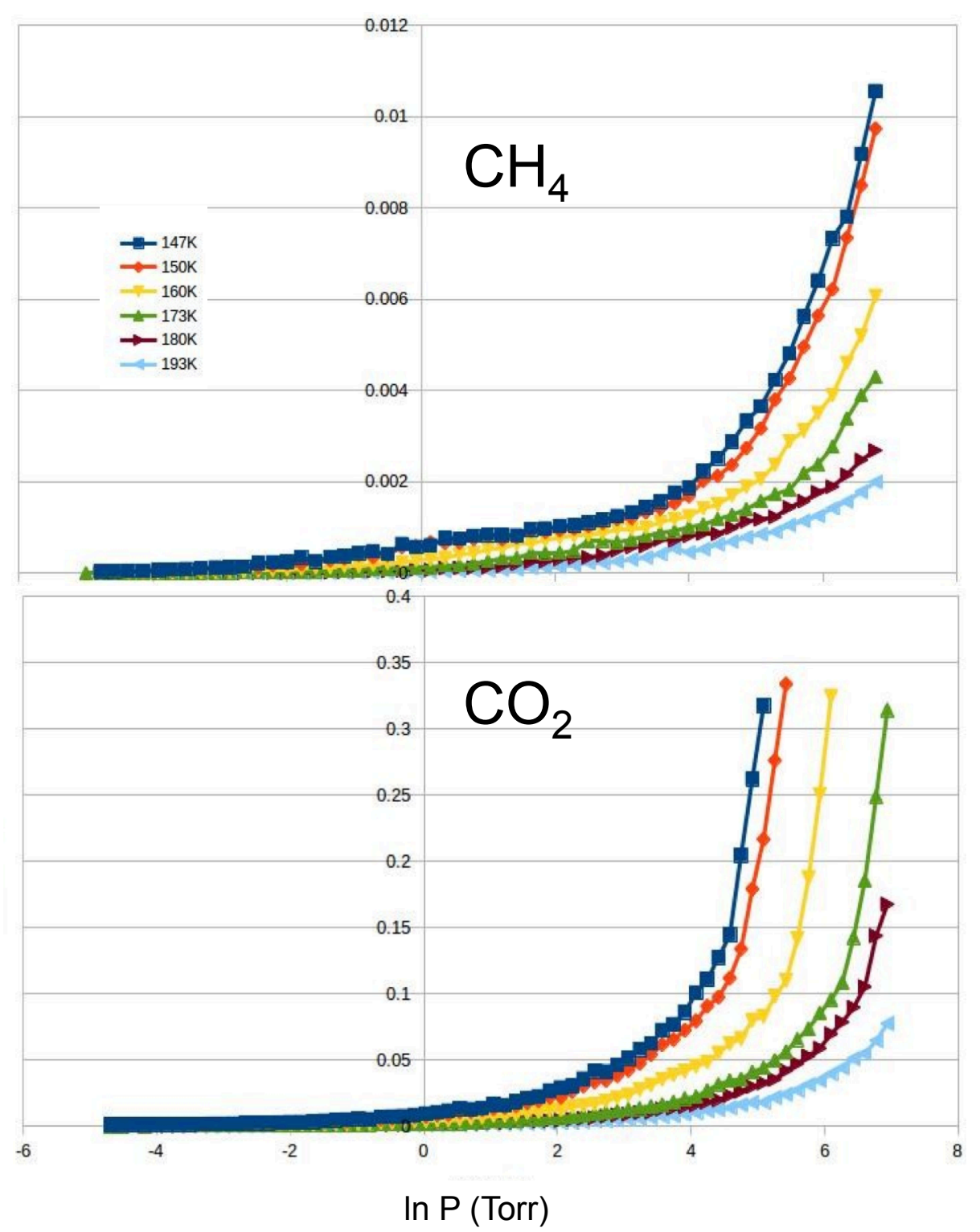

Figure 7. Adsorption isotherms (in arbitrary units) of methane (top) and carbon dioxide (bottom) at temperatures of 147, 150,160,173, 180 and $193 \mathrm{~K}$ from left to right. 


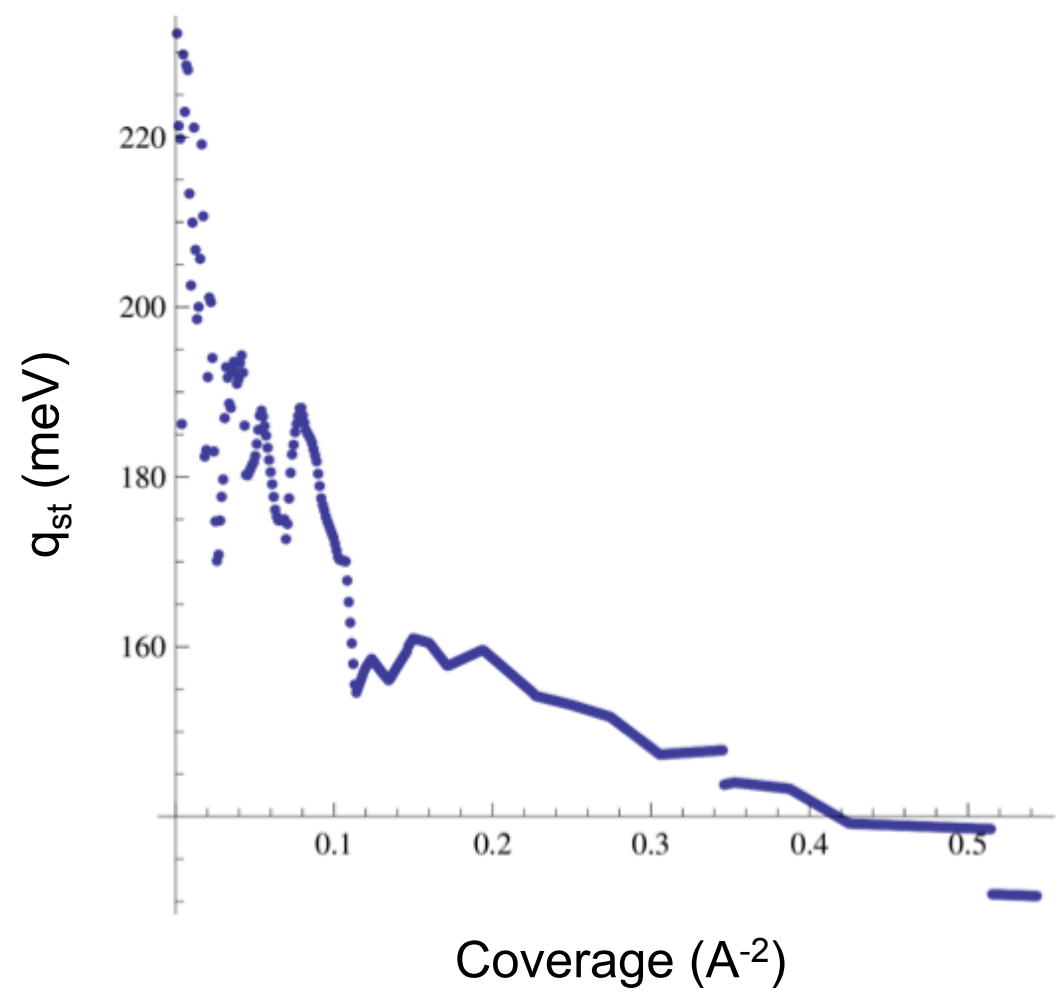

Figure 8. Isosteric heat of adsorption for $\mathrm{CO}_{2}$.

\section{Conclusions}

We have simulated the adsorption of neon, argon, carbon dioxide and methane in a simplified version of carbon nanohorns. Given the approximations made in our model, we have found close agreement with the experimental data available. The main simplification of our model is that there are only seven identical cones arranged on a flat lattice repeated periodically, while the real substrate is a spherical array of cones with different sizes and aspects.

The coincidences with the experimental data are many. For example, the isotherms of neon and argon feature bumps similar to those in the real substrate. Hence, we can conclude that those bumps observed in the experiment correspond to adsorption in the internal tip and interstices. The isotherms of carbon dioxide, on the contrary, are smooth, implying that internal and interstitial sites may have similar energies. The quantitative agreement is better for neon and argon than for $\mathrm{CO}_{2}$. One possible reason is that the adsorption of $\mathrm{Ne}$ and $\mathrm{Ar}$ is highly dominated by the two first sites, while for $\mathrm{CO}_{2}$, the external surface may be more significant. One of the simplifications of our model is that the substrate has only seven cones with three interstices; hence, the "exterior" is overexposed.

We also estimated, using the IAST, a high selectivity of $\mathrm{CO}_{2} / \mathrm{CH}_{4}$. A more precise calculation would require a heavier computation, either $\mathrm{MC}$ or $\mathrm{MD}$ of the $\mathrm{CO}_{2}+\mathrm{CH}_{4}$ mixture on a more realistic substrate. Our results can be used as a guide to the conditions of temperature and vapor pressure in which those calculations would be worth it.

Acknowledgments: S.G. and A.N. thank the financial support provided by the National Science Foundation, Partnership for Reduced Dimension Materials (PRDM), NSF Grant No. DMR1205608. S.G. thanks the financial support provided by the National Science Foundation Grant No. DMR-1006010 and DMR-1231319 . SG and AS thank the financial support provided by the National Science Foundation (Award No. NSF PHY-1358727) for the REU site in Physics at Howard University. We thank María M. Calbi and Aldo D. Migone for fruitful comments and discussions.

Author Contributions: A.S. ran the simulations and analyzed the data of neon and carbon dioxide. A.N. ran the simulations and analyzed the data of argon and methane. S.G. coordinated, supervised and wrote the article. 
Conflicts of Interest: The authors declare no conflict of interest.

\section{References}

1. Toigo. F.; Cole, M.W. Model adsorption potentials for He and Ne on graphite. Phys. Rev. B 1985, 32, 6989.

2. Boninsegni, M.; Cole, M.W.; Toigo, F. Helium adsorption on a Lithium substrate. Phys. Rev. Lett. 1999, 83, 2002.

3. Ancilotto, F.; Toigo, F. First-principles study of potassium adsorption on graphite. Phys. Rev. B Condens. Matter 1993, 47, 13713.

4. Calbi, M.; Toigo, F.; Cole, M.W. Dilation and intercalation of gases within carbon nanostructures. J. Low Temp. Phys. 2002, 126, 179-186.

5. Dillon, A.; Jones, K.; Bekkedahl, T.; Kiang, C.; Bethune, D.; Heben, M. Storage of hydrogen in single-walled carbon nanotubes. Nature 1997, 386, 377-379.

6. Chambers, A. ; Park, C.; Baker, R.; Rodriguez, N. Hydrogen storage in graphite nanofibers. J. Phys. Chem. B 1998, 102, 4253-4256.

7. Inoue, S.; Ichikuni, N.; Suzuki, T.; Uematsu, T.; Kaneko, K. Capillary condensation of N2 on multiwall carbon nanotubes. J. Phys. Chem. B 1998, 102, 4689-4692.

8. Teizer, W.; Hallock, R.; Dujardin, E.; Ebbesen, T. ${ }^{4}$ He Desorption from single wall carbon nanotube bundles: A one-dimensional adsorbate. Phys. Rev. Lett. 1999, 82 , 5305-5308.

9. Weber, S. ; Talapatra, S.; Journet, C.; Migone, A.D. Determination of the binding energy of methane on single-walled carbon nanotube bundles. Phys. Rev. B 2000, $61,13150$.

10. Pina-Salazar, E.Z.; Kaneko, K. Adsorption of water vapor on mesoporosity-controlled singe wall carbon nanohorn. Colloids Interface Sci. Commun. 2015, 5, 8-11.

11. Adelene Nisha, J.; Yudasaka, M.; Bandow, S.; Kokai, F.; Takahashi, K.; Iijima, S. Adsorption and catalytic properties of single-wall carbon nanohorns. Chem. Phys. Lett. 2000, 328, 381-386.

12. Calbi, M.; Cole, M.; Gatica, S.; Bojan, M.; Johnson, J.K. Adsorption by Carbons; Bottani, E., Tascon, J., Eds.; Elsevier Science: Amsterdam, The Netherlands, 2008; Chapter 9, 187-210.

13. Migone, A.D. Adsorption by Carbons; Bottani, E., Tascon, J., Eds.; Elsevier Science: Amsterdam, The Netherlands, 2008; Chapter 16, 403-430.

14. Gatica, S.; Calbi, M.; Diehl, R.; Cole, M. Review: Novel physics of gases near carbon nanotubes and buckyballs. J. Low Temp. Phys. 2008 152, 89-107.

15. Calbi, M.; Cole, M.; Gatica, S.; Bojan, M.; Stan, G. Colloquium: Condensed phases of gases inside nanotube bundles. Rev. Mod. Phys. 2001, 73, doi:10.1103/RevModPhys.73.857 .

16. Rols, S.; Johnson, M.; Zeppenfeld, P.; Bienfait, M.; Vilches, O.; Schneble, J. Argon adsorption in open-ended single-wall carbon nanotubes. Phys. Rev. B 2005, 71, 155411.

17. Stan, G.; Bojan, M.; Curtarolo, S.; Gatica, S.; Cole, M. Uptake of gases in bundles of carbon nanotubes. Phys. Rev. B 2000, 62, 2173-2180.

18. Gatica, S.; Bojan, M.; Stan, G.; Cole, M.J. Quasi-one- and two-dimensional transitions of gases adsorbed on nanotube bundles. Chem. Phys. 2001, 114, 3765-3769.

19. Krungleviciute, V.; Ziegler, C.A.; Banjara, S.R.; Yudasaka, M.; Iijima, S.; Migone, A.D. Neon and CO2 adsorption on open carbon nanohorns. Langmuir 2013, 29, 9388-9397.

20. Calvillo, A.; Russell, B.; Migone, A.; Iijima, S.; Yudasaka, M. Argon Adsorption on Open Carbon Nanohorns. Proceedings of APS March Meeting Abstracts, San Antonio, TX, USA, 2-6 March 2015; abstract Y16.002.

21. Krungleviciute, V.; Migone, A.D.; Yudasaka, M.; Iijima, S. CO2 Adsorption on Dahlia-Like Carbon Nanohorns: Isosteric Heat and Surface Area Measurements. J. Phys. Chem. C 2012, 116, 306-310.

22. Bansal, R.C.; Goyal, M. Activated Carbon Adsorption; CRC Press: Boca Raton, FL, USA, 2005.

23. Yang, R.T. Gas Separation by Adsorption Processes; Butterworths Publishers: Stoneham, MA, USA, 1987.

24. Maiga, S.M. Adsorption of Gases on Graphene and Metal Organic Frameworks. Ph.D. Thesis, Howard University, Washington, DC, USA, 2016.

25. Myers, A.L.; Prausnitz, J.M. Thermodynamics of mixed-gas adsorption. AIChE J. 1965, 11, 121-127.

26. Babarao, R.; Hu, Z.; Jiang, J.; Chempath, S.; Sandler, S.I. Storage and separation of $\mathrm{CO}_{2}$ and $\mathrm{CH}_{4}$ in silicalite, C168 schwarzite, and IRMOF-1: A comparative study from Monte Carlo simulation. Langmuir 2007, 23, 659-666. 
27. Iijima, S.; Yudasaka, M.; Yamada, R.; Bandow, S.; Suenaga, K.; Kokai, F.; Takahashi, K. Nano-aggregates of single-walled graphitic carbon nano-horns. Chem. Phys. Lett. 1999, 309, 165-170.

28. Frenkel, D.; Smit, B. Understanding Molecular Simulation; Academic Press Inc.: Orlando, FL, USA, 2001.

29. Harris, J.G.; Yung, K.H.J. Carbon Dioxide's Liquid-Vapor Coexistence Curve And Critical Properties as Predicted by a Simple Molecular Model. Phys. Chem. 1995, 99, 12021-12024.

30. Jiang, J.W.; Sandler, S.I.J. Separation of CO2 and N2 by Adsorption in C128 Schwarzite: A Combination of Quantum Mechanics and Molecular Simulation Study. J. Am. Chem. Soc. 2005, 127, 11989-11997.

31. Bruch, L.; Cole, M.; Zaremba, E. Physical Adsorption: Forces and Phenomena ; Dover Publications: Mineola, NY, USA, 2007.

32. Berry, R.S.; Rice, S.A.; Ross, J. Physicalchemistry; John Wiley: NewYork, NY, USA, 1980.

33. Watts, R.O.; McGee, I.J. Liquid State Chemical Physics; Wiley: New York, NY, USA, 1976.

34. Maitland, G.C.; Rigby, M.; Smith, G.C.; Wakeham, W.A. Intermolecular Forces: Their Origin and Determination; Clarendon: Oxford, UK, 1987; Tables A 3.1 and A 3.2, pp. 565-566.

Sample Availability: Not available.

(C) 2016 by the authors; licensee MDPI, Basel, Switzerland. This article is an open access article distributed under the terms and conditions of the Creative Commons Attribution (CC-BY) license (http://creativecommons.org/licenses/by/4.0/). 